\title{
DEVELOPMENT OF AN ICT INFRASTRUCTURE FOR INTERNET BASED INTEGRATION
}

\author{
John P.T. Mo \\ CSIRO,John.Mo@csiro.au \\ Ron C. Beckett \\ Reinvention Network,rcb@reinvent.net.au \\ Laszlo Nemes \\ CSIRO,Laszlo.Nemes@csiro.au \\ Stuart Woodman \\ CSIRO,StuartWoodman@csiro.au \\ AUSTRALIA
}

\begin{abstract}
Information Communication Technology (ICT) infrastructures supporting complex operations require collaborative effort to integrate a range of standalone software products into a coordinated system. However, the standalone products are normally developed with specific objectives and do not interoperate with each other. In a virtual enterprise environment, this imposes major challenges to ICT managers to manage a large variety of software products whereby each product only satisfies the unique requirements of a small portion of the user community. This paper discusses the development of the ICT infrastructure for a tooling virtual enterprise) using the open protocol standards as well as a new ICT infrastructure development tool known as Dynamic Network System (DNS). DNS models the operating characteristics of the ICT environment and generates the integrated internet web portal quickly from the system designer's view. It is built on standard JSP server capabilities using open source system that has the normal server security facilities.
\end{abstract}

\section{INTRODUCTION}

More than ever, global market conditions are characterised by frequent changes in products, services, processes, organisations, markets, supply and distribution networks (McNamara, 2003). Organisations need to be highly flexible in confronting this business environment (Nemes \& Mo, 2004). They may form a temporary alliance to deliver a project or product which they dissolve when the job is completed. The teams work together as an entity for a goal but the relationships among themselves and the individual companies they come from often rely on trust and industry practices (Hao et al, 2005).

With the globalisation of manufacturing activities, it is inevitable that more and more companies adopt a new business model for managing the complete product life cycle of manufacturing from product design to after sales services (Van den Berg \& Tolle, 2000). This type of temporary alliance is commonly known as a virtual 
enterprise (VE). Success for achieving the goal therefore demands well-coordinated agility in all internal and external aspects of the virtual enterprise. An effective ICT infrastructure can support the day-to-day operations of geographically separated partners (Shinonome et al, 1998).

Experience has shown that companies are willing to invest in improvements to their communication networks provided that there are clear benefits in return (Barrads \& Pinto-Ferreira, 2004). Unfortunately, without proper understanding of the fundamentals in a digital virtual enterprise, company alliances seem to be trying to solve their problems in an ad hoc fashion. Custom tailored solutions are very costly, at the level of several million dollars, not only for the collaborative system, but also for resource management, training, team building, document management and constant modifications due to the ever-changing business process environment (Ortiz et al, 1999). The business world needs to have the capability to set up their collaborative system supporting the VE in a very short time frame without making costly mistakes.

Information Communication Technology (ICT) infrastructures supporting complex operations such as ship building, tool making, automobile manufacturing and utilities management require collaborative effort to integrate a range of standalone software products into a coordinated system (Jiang \& Mo, 2001). However, the standalone products are designed with specific objectives and do not interoperate with other software products in an open computing environment in which many networks are interlinked. The challenge for many ICT managers is to manage a large variety of software products that each of them only satisfies the unique requirements of a small portion of the user community.

This paper discusses the development of an ICT infrastructure on which an internet based integration platform can be implemented. The platform, known as Dynamic Network System (DNS), uses a model driven concept which greatly reduces the need to re-program as the VE changes to different phases and entities in its life cycle.

\section{COMMUNICATION FRAMEWORK FOR VE}

In Australia, and in many other countries, large manufacturing firms are moving to countries with lower labour rates. Small contract manufacturing or tool making firms have traditionally been part of a vertically integrated supply chain where they were linked with product manufacturers and distributors. As these client companies move production offshore, or as they seek to reduce their internal costs by dealing with fewer suppliers, linkages with the small companies are broken.

An initiative called RELINK is investigating how small firms in the tooling and automotive industry can draw on innovations that are available to enable them to participate as part of a broader global supply chain. It is based on the establishment of large-scale virtual organizations of collaborating firms linked by a common vision and set of business practices that are supported by ICT tools.

RELINK is a joint initiative of two industry associations in Australia: FAPM (Federation of Automotive Products Manufacturers) and TIFA (Tooling Industry Forum of Australia). To understand how the formation of virtual enterprise relates to ICT infrastructure, a 5 layer model, RELINK Communication Framework (RCF), 
is used to describe the functionalities required to carry out the tasks of transmitting information from one end to another (Mo et al, 2005).

RCF is an open ICT infrastructure model that aims to support implementation of systems for the virtual enterprise at the appropriate level. The 5 layers are:

- Application layer: describes the functions that a toolmaker needs to do their work.

- Presentation layer: converts the information to a format suitable to be used by the application that the toolmaker wants to run.

- Interpretation layer: performs the function of diverting information into relevant streams.

- Verification layer: performs the function of ensuring the information transferred is actually the data that the sender wants to transmit.

- Physical layer: represents the transmission media that will be used in the communication process.

By characterising the communication profiles in RCF, it is observed that the ICT requirements of a VE change over time at different stages of the virtual enterprise development. To understand this phenomenon, the Virtual Enterprise Reference Architecture (VERA) (Zwegers et al, 2003) was used to provide a framework for analysis. VERA conforms to the Generalised Enterprise Reference Architecture and Methodology (GERAM) (IFIP-IFAC Task Force, 1999). It provides a unified framework for knowledge integration in distributed environment. The high level modelling framework facilitates identification of processes but it does not address system implementation issues such as security or quality of service.

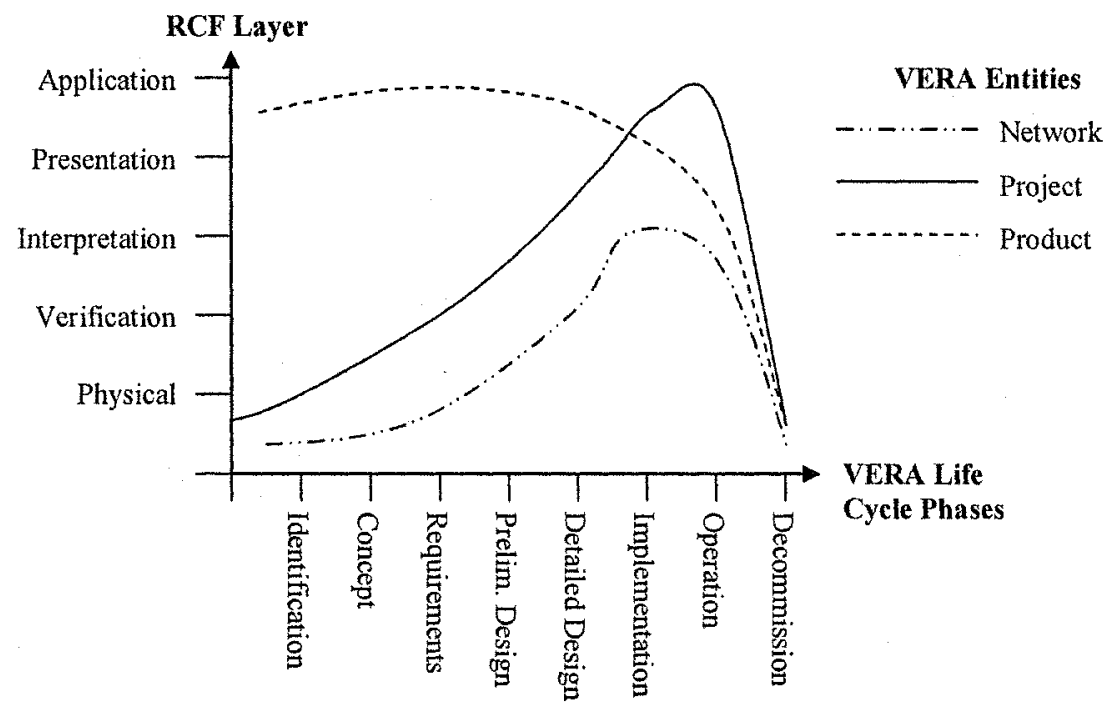

Figure 2 ICT profiles in VERA

The ability of the companies to quickly align their objectives and information systems with the amalgamated business processes can benefit the VE by supporting effective decision-making and informed actions. Without the right information at 
the right time, delays and more seriously costly mistakes can occur. VERA enables modelling of the relationship between the changes of ICT requirements to the maturity of the virtual enterprise, i.e. the life cycle phases in VERA.

In Figure 2, the Network entity represents the start of the virtual enterprise. There is very little demand for ICT support. Person to person relationships and trust are more important aspects towards the building of the entity. Hence, the ICT profile for the Network entity remains low until the point where the partners start to exchange documentation, which are the detailed design and implementation phases. Once the consortium is confirmed, the virtual enterprise changes to a Project entity.

The ICT profile for the Project entity increases quickly at lower RCF levels from the initial conceptual phases to highly sophisticated presentation and application levels as the entity progresses to the detailed design, implementation and operation phases. In these phases, large amount of data including design, production schedule, costing and legal documentations are exchanged.

The ICT profile remains at the high level as the Product (i.e. the tool) information is progressively refined during manufacturing. However, as described in the scenario in Figure 2, once production of the tool starts, the need to exchange complicated data sets reduces. The ICT levels become lower as the Product entity approaches completion.

\section{EXPERIENCE ON APPLICATION SOFTWARE}

There are many criteria for benchmarking ICT capabilities in an organisation (Shen et al, 2004). The ever-changing needs of the VE indicate that the criteria for an ICT infrastructure supporting complete VERA life cycle operation must be open to interoperate with pre-existing software as well as flexible with high adaptability to different levels of sophistication (Aerts et al, 2000). As part of the RELINK study, a range of relatively simple commercial ICT tools used by the tooling industry are evaluated against the criteria for supporting establishment of the VE.

\subsection{Microsoft Project}

It is highly sophisticated software with a large number of functions that many people have difficulty in understanding it. By way of example, a textbook available to help explain the operation of Project 2003 contains about 900 pages. The underlying principle of MS Project is PERT (Program Evaluation and Review Technique). The integration of different views of the same project information is the most important feature that it can offer. However, it is a standalone software and does not have the ability to share with partners without total transfer of the project model. There are also severe restrictions on the conceptual framework that makes it difficult to be used for managing VE activities, for example, the segmentation of tasks over a long period with intermittent operating requirement is not sufficiently handled. 


\subsection{Microsoft Project Server}

It is marketed as an internet enabled version of MS Project. However, installation of MS Project Server is troublesome. It requires many software components that are not normally required in day to day computing. These include:

- Microsoft Windows Server 2003

- Microsoft SQL Server

- Microsoft Sharepoint

- IIS

These additional system components add another level of complexity and costs in the ICT infrastructure. As each of the above software components are expensive by themselves, the initialisation cost is almost 4 times more than MS Project itself. It was also noted that management of the infrastructure would require a full time IT staff, which many VEs are not prepared for when they are formed.

\subsection{Outlook Express}

It is common software that is available to everyone who has Windows based PCs. However, it is not interoperable with other operating systems such as Sun's Solaris, which is commonly used for professional CAD/CAM environments. Outlook has good network connectivity and can be used for email if the server it connects to supports Post-office protocol (POP3). In that regard, Outlook can be classified as supporting the Verification Level of the RCF.

\subsection{Quickplace}

It is a web-based software running on PC server to support web-based collaborative work. The interface is relatively easy to use but the functionality offered is rather limited. Significant enhancement in the form of specially written Java software modules is required to introduce abilities such as automatic message filing and directory changes.

\subsection{KickStart}

It is simple, low cost project management software that uses the MS Project concept but does not have all the sophistication. However, its capability is therefore limited on a PC based environment. Linkage to Outlook tasks can be enabled.

\subsection{PHP Bulletin Board}

The bulletin board is internet-based discussion software that captures the discussion of participants in a structural fashion. It runs on many platforms and can be accessed via an internet browser. The bulletin board is best used for idea exchange, knowledge search, and problem solving involving expert's interaction on a distributed environment. The problem of bulletin board is that although a data structure can be established easily, conformant to the data structure is highly dependent on the users.

\section{DYNAMIC NETWORK SYSTEM}

The software evaluation result shows that commercial ICT tools used by the toolmakers do not interoperate easily. This issue is well recognised and proved to 
be the bottleneck in fostering collaboration between companies (Kosanke, 2005). Solutions that are based on open distributed environment are of particular attraction to SMEs, which have limited ICT support resources to adopt full scale interenterprise collaboration systems (Davidsson et al, 2005). An added problem with ICT support for the RELINK type organisation is that at different states of the virtual enterprise, the requirements for ICT support change in time. Companies involved in the virtual enterprise find it difficult to optimise their ICT investments in the most cost effective fashion. A flexible approach that facilitates the detailed design of ICT systems and their rapid implementation and ongoing development is required. Systems that are component based and built on systems engineering approaches are more likely to meet the specification for large scale, change-capable enterprise systems (Weston, 1999).

The development of the Dynamic Network System (DNS) has the objectives of being highly flexible for adaptation to the VERA life cycle as well as integrating pre-existing ICT capabilities of the members of the VE into the collaborative environment with minimum cost requirements. DNS achieves these objectives by combining two conceptual designs. First, DNS models the operating characteristics of the ICT environment and can be used to generate the integrated internet web portal quickly from the system designer's view. Second, DNS is built on open source Java server pages (JSP) server capabilities and standard TCP/IP RMI technologies. The server security and management capabilities are interoperable with any other normal web servers. DNS does not provide specific enterprise functions by itself, but rather it is a concept capturing platform that incorporates third party software tools with those enterprise functions into an integrated information system. The underlying computing environment then manages the execution of the software tools which produce processed information passed on by the DNS model to the next software tool.

DNS software architecture can be depicted in Figure 3. An Apache server provides the web interface of the system to outside world. Enhanced by Tomcat, an open source server, development of DNS modules becomes a much easier project as most of the internal controls are already built in. Installation of Apache and Tomcat is easy and instructions can be found at http://tomcatapache.org. DNS can be divided into two main parts: DNS Modeller and DNS RunTime.

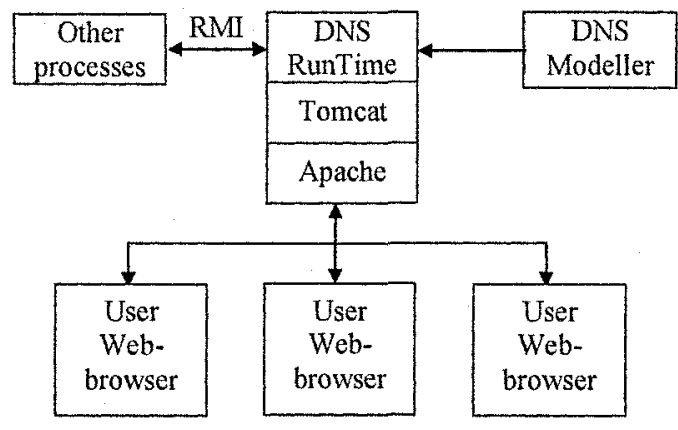

Figure 3 DNS software architecture 
DNS performs its functions by calling operational components known as agents. An agent is a Java class that exhibits certain operational behaviour and so it is also known as behavioural class. Basic functionality such as displaying information and setting up user selection pages are included as built in agents in the system. Additional agents can be incorporated into the system easily by the user with his/her own Java classes. In essence, each agent must be "extended" from a class au.csiro.dns.behaviour.BehaviourClass, which is included in the DNS distribution jar file. When the user's agent is created, the user can call upon this behaviour in the DNS model after a simple registration procedure to DNS RunTime.

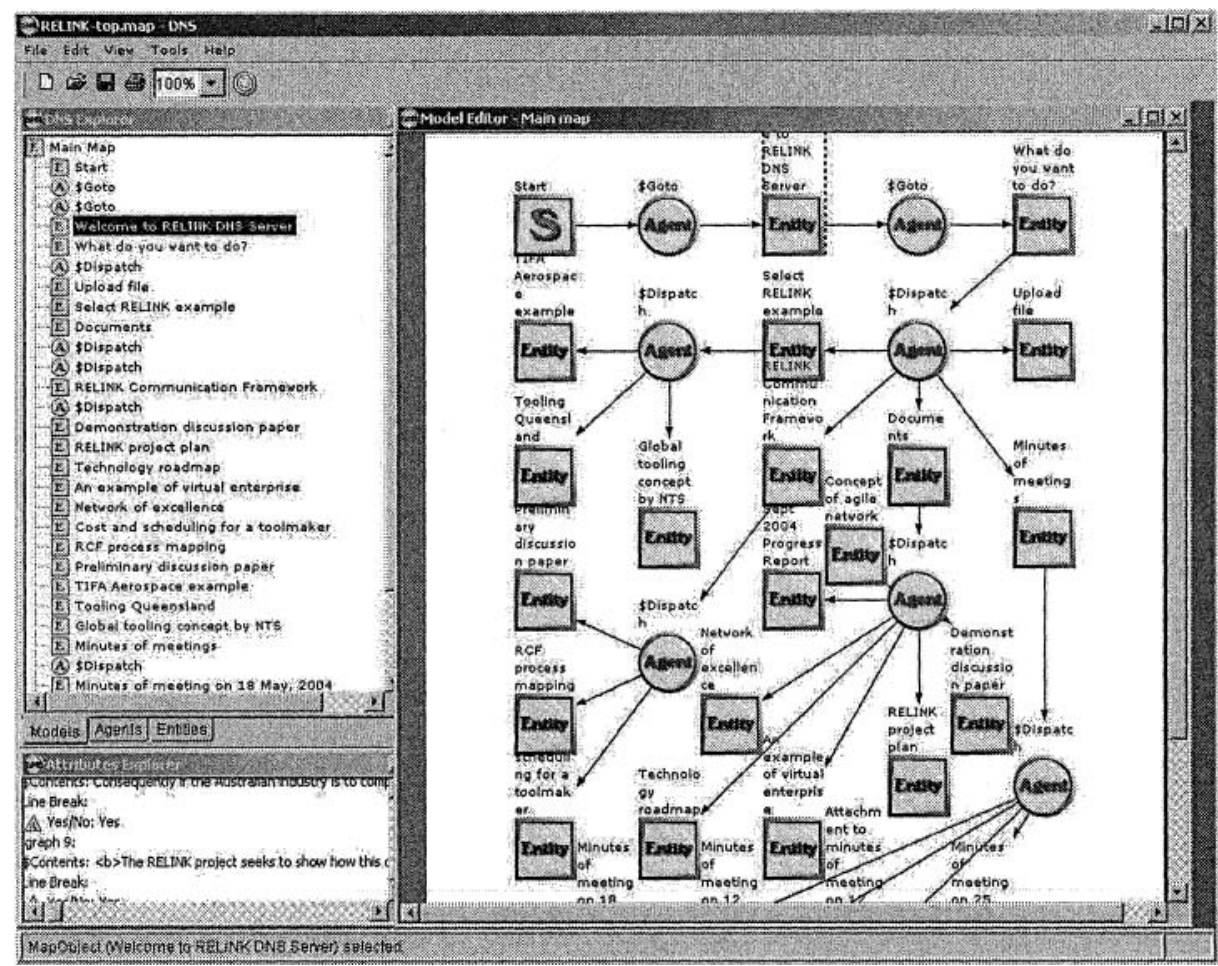

Figure 4 DNS Modeller user interface

DNS operates according to a defined DNS model, which is created by the DNS Modeller. The DNS Modeller is the user interface for modelling the functionality and behaviour of the collaborative system. The user interface is shown in Figure 4.

DNS modelling concept consists of two main components. The agent entity will deliver operational characteristics of the system when it is executed. An agent's behaviour will result in certain state of system known as entity. Hence, a DNS model captures the operational details of a remote user interacting with the system to achieve his/her goals. Since DNS can incorporate agents developed by any user supplied Java class, the system is highly flexible in exhibiting various desirable server capabilities through a wide variety of agents registered to it. The outcome of running the DNS Modeller is a DNS model that captures the anticipated interactive processes of the web-based collaborative system. 
The DNS RunTime runs in conjunction with Tomcat and translates the DNS model into a governing framework. When the DNS model is activated through DNS RunTime, the information is translated into web server information and drives the Tomcat and Apache system to deliver the functionality to the web user (Figure 5). DNS uses standard internet server technologies with functionalities added on separately as needed. For example, knowledge is better managed by a structured directory that can be browsed by the users. Experience shows that a pre-agreed directory structure is necessary to gain acceptance by all VE partners. Since the agreed structure represents an alignment of the practices of individual companies to the VE, users of the system can find the mutually accepted knowledge repository easy to adapt.

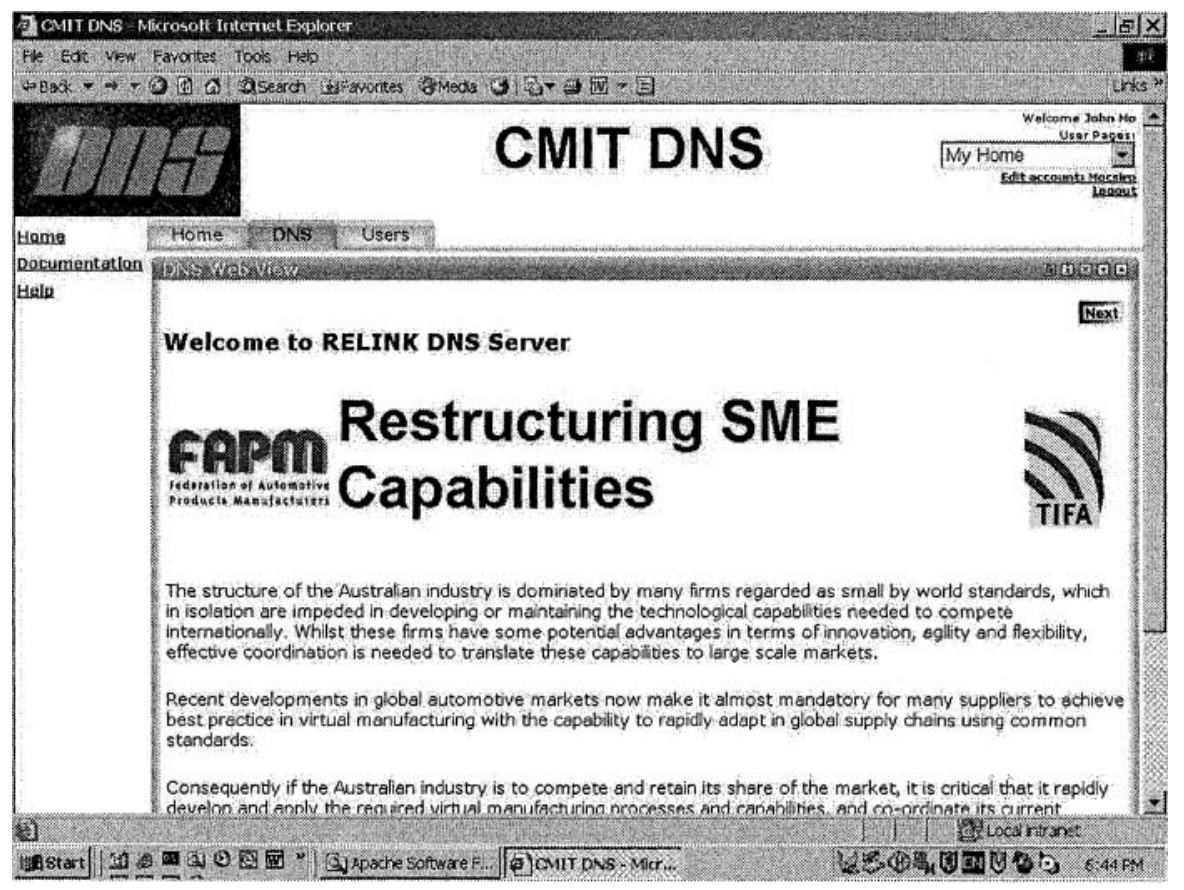

Figure 5 DNS RunTime driven web server

Another example is the automatic processing of documents in the system. File downloading is an integral part of the server capability. Suitable links can be inserted easily in the text that can be activated to download files and data. File uploading is a separate feature that must be implemented using some server programming facilities. The JSP capability can be easily implemented from standard function library. Other functionalities such as bulletin board and email can also be incorporated in the open ICT infrastructure.

In addition to standard functionalities, DNS can integrate third party collaborative functionality using RMI. Interfacing agents that work with other systems such as invoking a third party software can be implemented allowing it to manage the interaction with third party processes that may need to be called upon 
during execution of the collaborative task. For example, information searching processes can be linked by DNS via a RMI enabled Java program that manages its own Java database connectivity (JDBC) with any SQL database. In DNS Modeller, the RMI behavioural class is defined in the same way as a normal agent.

\section{CONCLUSION}

This paper evaluated the usefulness of implementing and using a number of common server functionalities for supporting VERA life cycle. These functions are supported by capabilities that come as an integral part of the open source server. The development of DNS leads to a flexible and open platform for integrating preexisting ICT infrastructures in VE partners.

Since the system requires open source server components and industry standard JSP technologies, the cost and development effort required for developing a collaborative server is low. Management of the system does not require substantial IT skills and is ideal for SMEs.

Recent developments in internet based technologies on open protocol standards such as SOAP have provided an opportunity to develop an integrated internet-based system while maintaining the autonomy of individual software products in their normal operating environments. Due to the complexity of the application, these open standards require highly skilled professionals who are well trained in the fundamental working principles of the standards as well as system diagnosis. To enable a more general use of the new internet based technologies, a simplified process and methodology is required. It is anticipated that by application of the latest distributed computing technologies, further development of DNS will be directed towards a multi-platform integrated environment.

\section{REFERENCES}

1. Aerts A.T.M., Szirbik N.B., Goossenaerts J.B.M., (2000), "Flexible Infrastructure for Virtual Enterprises", in Global Engineering, Manufacturing and Enterprise Networks, ed. Mo J.P.T., Nemes L, IFIP TC5 WG5.3/5.7/5.12 Fourth International Working Conference on the Design of Information Infrastructure Systems for Manufacturing (DIISM 2000), November 15-17, Melbourne, Australia, pp.26-37

2. Barradas L.C.S., Pinto-Ferreira J.J., "P2P Infrastructure for tourism electronic marketplace", Paper $49,18^{\text {th }}$ IFIP World Computer Congress, Toulouse, France, 22-27 August, 2004

3. Davidsson P., Ramstedt L., Tornquíst J. (2005). "Inter-organisation Interoperability in Transport Chains Using Adapters Based on Open Source Freeware", Proceedings of the First International Conference on Interoperability of Enterprise Software and Applications, INTEROP-ESA'05, 22-25 February, Geneva, Switzerland

4. Hao Q., Shen W., Wang L., (2005). "Towards a cooperative distributed manufacturing management framework ${ }^{20}$, Computers in Industry, 56 (2005) 71-84

5. IFIP-IFAC Task Force on Architectures on Enterprise Integration (1999). "Generalised Enterprise Reference Architecture and Methodology", Annex to ISO WD15704, Requirements for enterprise reference architectures and methodologies, GERAM v.1.6.3, March

6. Jiang H.C., Mo J.P.T., (2001). "Internet Based Design System for Globally Distributed Concurrent Engineering", Journal of Cybernetics and Systems, Vol.32, No.7, October-November, pp.737 754

7. Kosanke K. (2005). "ISO Standards for Interoperability: a comparison", Proceedings of the First International Conference on Interoperability of Enterprise Software and Applications, INTEROPESA'05, 22-25 February, Geneva, Switzerland 
8. McNamara, D.L (2003) "Scale, Space and Place - SME Flexibility in Cross-border Industrial Clusters" Asia-Pacific Researchers in Organisational Studies, Conference Proceedings, Oaxaca, Mexico, December 8-10

9. Mo J.P.T., Beckett R., Nemes L., (2005). "Technology Infrastructure for Virtual Organisation of Tooling", Sixth IFIP Working Conference on Virtual Enterprises (PRO-VE'05), 26-28 September, 2005, Valencia, Spain

10. Nemes L., Mo J.P.T., (2004), "Collaborative Networks in Australia - Challenges and Recommendations", in Collaborative Networked Organizations, ed. Camarinha-Matos L.M., Afsarmanesh H., pub. Kluwer Academic Publishers, ISBN 1-4020-7823-4, pp.97-102

11. Ortiz A., Lario F., Ros L. (1999), "Enterprise Integration - Business Processes Integrated Management: a proposal for a methodology to develop Enterprise Integration Programs", Computers in Industry, 40, 155-171

12. Shen H., Wall B., Zaremba M., Chen Y.L., Browne J., (2004), Integration of business modelling methods for enterprise information system analysis and user requirements gathering, Computers in Industry, 54 (2004), 307-323

13. Shinonome M., Hashimoto H., Fuse A., Mo J.P.T., (1998). "Development of an information technology infrastructure for extended enterprise", IFIP TC5 WG5.3/5.7 Third International Conference on the Design of Information Infrastructure Systems for Manufacturing (DIISM '98), May 18-20, Fort Worth, Texas, U.S.A., pp.353-364

14. Van den Berg R.J., Tolle M., (2000). "Assessing Ability to Execute in Virtual Enterprises", in Global Engineering, Manufacturing and Enterprise Networks, ed. Mo J.P.T., Nemes L., IFIP TC5 WG5.3/5.7/5.12 Fourth International Working Conference on the Design of Information Infrastructure Systems for Manufacturing (DIISM 2000), November 15-17, Melbourne, Australia, pp. $38-45$

15. Weston R.H., (1999), "Reconfigurable, component-based systems and the role of enterprise engineering concepts", Computers in Industry, 40, pp.321-343

16. Zwegers A., Tolle M., Vesterager J., (2003). "VERAM: Virtual Enterprise Reference Architecture and Methodology", in Global Engineering and Manufacturing in Enterprise Networks, ed. Karvonen I., van den Berg R., Bernus P., Fukuda Y., Hannus M., Hartel L., Vesterager J., VTT Symposium 224, Helsinki, Finland, 9-10 December, pp.17-38 